\title{
Travel and immunosuppressant medication
}

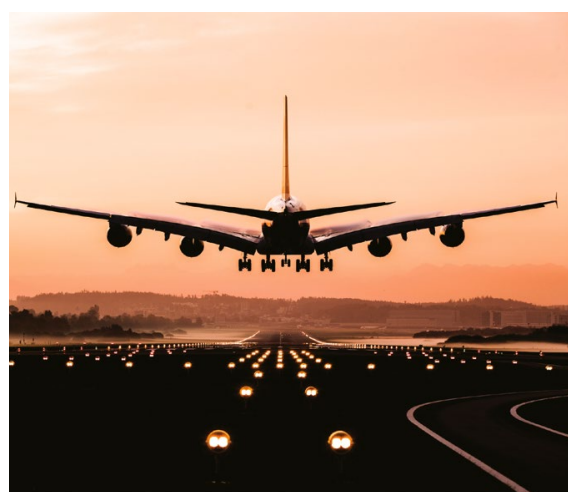

CPD

\section{Nicholas A Zwar}

\section{Background}

The use of potent immunosuppressant medications is becoming more common, particularly the use of biologicals for a range of conditions such as rheumatoid arthritis and inflammatory bowel disease.

\section{Objective}

This article focuses on the implications of immunosuppressant medications for travel, including pre-travel vaccinations, minimising risks during travel and travelling with medicines.

\section{Discussion}

Pre-travel risk assessment is essential to prepare for safe travel. Live vaccines are contraindicated in people with significant immunosuppression because of the higher risk of adverse events and vaccine-associated disease. Inactivated vaccines can be used but may be less effective. Assessing the degree of immunocompromise in patients taking immunosuppressants includes considering both the medications and the underlying conditions. An individualised approach, often involving expert input, is needed to provide pre-travel health advice and immunisation. Planning ahead for travel is needed to minimise risks.
MORE THAN EVER BEFORE, people who are immunocompromised are wanting to travel. In the USA, it is estimated that people who are immunocompromised comprise $1-2 \%$ of patients seen in travel clinics, ${ }^{1}$ and this is likely to increase with widening indications for immunosuppressant medications. Immune suppression may be caused by a medical condition such as human immunodeficiency virus (HIV), by medication (eg steroids and biological agents) or by treatments (eg radiotherapy). There are multiple reasons for the increase in the number of people with immunosuppression who are travelling. These reasons include more older people and those with long-term conditions travelling, the advent of highly effective antiretroviral medications that have transformed HIV into a long-term condition, and the increasing use of tumour necrosis factor (TNF) and other biological agents for a range of diseases including inflammatory bowel disease, autoimmune inflammatory rheumatic diseases and skin conditions such as psoriasis.

This article focuses on the implications of immunosuppressant medications for travel and does not cover the effects of underlying diseases such as HIV, malignancies including leukaemia and lymphoma, aplastic anaemia, anatomical or functional asplenia, multiple sclerosis, graft versus host disease, renal disease and congenital immune deficiencies. It should be noted that such patients can be immunocompromised even when not receiving treatment, and caution and a collaborative approach involving other members of their treating team will be needed if travel is planned. Case-by-case advice from the oncology, rheumatology or immunology team may need to be sought.

\section{Pre-travel health advice}

All travellers would benefit from pre-travel health advice, but it is particularly necessary for those who are taking immunosuppressant medication. Ideally this group would seek advice at least six weeks before departure. A defining feature of travel medicine is risk assessment, which involves a thorough evaluation of the traveller's health status and medical and vaccination history, destinationspecific risks including the epidemiology of infectious diseases in the region of travel and itinerary-specific risks including the length of travel, type of accommodation, urban versus rural travel, modes of transport and higher-risk activities. ${ }^{2,3}$ As well as advice about infectious diseases, including vaccine-preventable disease, 
travellers need advice on food and water hygiene, mosquito avoidance, avoidance of animal bites, safety and security and any destination-specific risks. Travellers who have significant medical conditions should carry a health summary, including a list of current medications and any allergies.

Live vaccines pose particular risks of adverse effects and vaccinerelated disease in patients who are immunocompromised. Live vaccines include Bacillus Calmette-Guérin (BCG); live attenuated influenza vaccine; yellow fever vaccine; herpes zoster vaccine; varicella vaccine; measles, mumps and rubella vaccine; rotavirus vaccine; live attenuated Japanese encephalitis vaccine and oral typhoid vaccine. Oral polio vaccine is also a live attenuated vaccine but is no longer available in Australia. The vaccines most likely to lead to vaccinerelated disease caused by unchecked infection (replication) by the live vaccine virus or bacteria are BCG and vaccines that contain measles, mumps, rubella or varicella-zoster virus. ${ }^{4}$ Inactivated vaccines do not have an increased risk of adverse effects for people who are immunocompromised but may be less effective. ${ }^{1,4}$

For people who are severely immunocompromised and planning travel to high-risk destinations, the advice sometimes needs to be not to travel or to significantly modify the itinerary. In the pre-travel consultation context, this issue most commonly arises when people plan to travel to areas of yellow fever risk. Yellow fever vaccine is contraindicated in people with significant immunosuppression as there is a higher risk of serious adverse effects including both viscerotropic and neurotropic reactions. ${ }^{1}$ These severe adverse reactions can be fatal, as viscerotropic adverse effects can lead to multiple organ failure. If a patient who is immunocompromised decides to travel to a yellow fever risk area, then a yellow fever vaccination waiver letter can be provided, but the patient needs to be aware that they are at risk of severe disease and death if exposed when unvaccinated.

As the most common source of pre-travel medical advice, ${ }^{5}$ the general practitioner (GP) will be the starting point for pre-travel health advice for most patients. The patient's regular GP will also have knowledge of the underlying condition, comorbidities and vaccination history. However, advice varies depending on the underlying condition, immunosuppressant medication, travel risks and particular vaccines indicated. Given this complexity, a team approach is often needed involving the GP, the treating specialist or an immunologist and, depending on the GP's degree of expertise, a specialised travel medicine clinic. It is important to adequately document the advice given.

\section{Assessing effects of medication on immune status}

The first step is to assess the reasons for and extent of the traveller's immune compromise. One of the most commonly used groups of medications that can cause immune compromise are corticosteroids. The nature of the underlying condition needs to be considered, but according to the US Centers for Disease Control and Prevention (CDC), ${ }^{1}$ people are generally not significantly immunocompromised if they are receiving:

- short- or long-term daily or alternating day doses of $<20 \mathrm{mg}$ prednisolone or equivalent

- inhaled or topical steroids

- steroid injections (eg intra-articular)

- physiological doses of maintenance steroids.

Patients are considered immunocompetent if more than one month has passed since the end of a course of high-dose steroid (>20 mg prednisolone for $>2$ weeks).

Particular care is needed when steroids are being taken with other medications that affect the immune system, and non-GP specialist advice will often be needed.

Some medications are highly likely to cause severe immunocompromise. According to both The Australian immunisation handbook ${ }^{4}$ and the CDC, ${ }^{1}$ people taking any of the following categories of medication are considered severely immunocompromised:

- High-dose corticosteroids, defined as $\geq 2 \mathrm{mg} / \mathrm{kg}$ per day ( $20 \mathrm{mg}$ ) of prednisone or equivalent in people who weigh $>10 \mathrm{~kg}$, when administered for $\geq 2$ weeks. These patients should not receive a live vaccine for $\geq 1$ month after discontinuation of high-dose systemic corticosteroids.

- Alkylating agents such as cyclophosphamide.

- Antimetabolites such as azathioprine, 6-mercaptopurine and methotrexate. However, at usual doses (methotrexate $\leq 0.4 \mathrm{mg} / \mathrm{kg} /$ week, azathioprine $\leq 3 \mathrm{mg} / \mathrm{kg} /$ day, or 6-mercaptopurine $\leq 1.5 \mathrm{mg} / \mathrm{kg} /$ day), it is generally considered safe to use either the live attenuated herpes zoster vaccine or the recently approved inactivated vaccine. According to The Australian immunisation handbook, ${ }^{4}$ this includes people $\geq 50$ years of age who are also taking low-dose corticosteroids (<20 mg per day of prednisoneequivalent dose).

- Transplant-related immunosuppressive medications including cyclosporine and tacrolimus.

- Cancer chemotherapeutic agents.

- Tumour necrosis factor blockers such as etanercept, adalimumab, certolizumab pegol, golimumab and infliximab.

- Other biological agents, particularly lymphocyte-depleting agents (thymoglobulin or alemtuzumab) and B cell-depleting agents (rituximab). There are a number of older diseasemodifying antirheumatic medications (sulfasalazine, hydroxychloroquine) that do not cause immune suppression at usual doses.

The Australian immunisation handbook recommendations for live vaccines and corticosteroids are presented in Table 1. The advice from the UK is somewhat different. ${ }^{6}$ UK recommendations are that live vaccines are contraindicated in travellers for three months after the cessation of high-dose $(2 \mathrm{mg} / \mathrm{kg}$ or $\geq 40 \mathrm{mg}$ per day for $\geq 7$ days, or $1 \mathrm{mg} / \mathrm{kg}$ /day for one month) oral or rectal corticosteroids for conditions other than adrenal insufficiency.

\section{Immunosuppressive medication} and inactivated vaccines

Immunosuppressive medications can also have an effect on the immune response to inactivated vaccines. According to the 
CDC, ${ }^{1}$ if doses of inactivated vaccines are given within two weeks before or while receiving medications causing severe immunocompromise, they should be regarded as ineffective and not counted towards a primary vaccination schedule. There are some exceptions; for example, a response to hepatitis $\mathrm{A}$, influenza and pneumococcal vaccines is seen in patients taking TNF blockers.
There are considerations for specific vaccines, as shown in Table 2.

\section{Immunosuppressant medication} and other risks during travel

People who are immunosuppressed either because of medications or underlying disease, or a combination of both, are at higher risk of severe or chronic enteric infections. Great care with sanitation, particularly hand washing, and food and fluid choices is needed. Providing antibiotics for self-treatment of traveller's diarrhoea should be considered, but there is a potential for medication interactions among patients already taking medications for chronic medical conditions. Malaria prevention advice and choice of prophylaxis are similar to other travellers, but

\section{Table 1. Recommended timing of live vaccine doses in adults and children taking corticosteroids*}

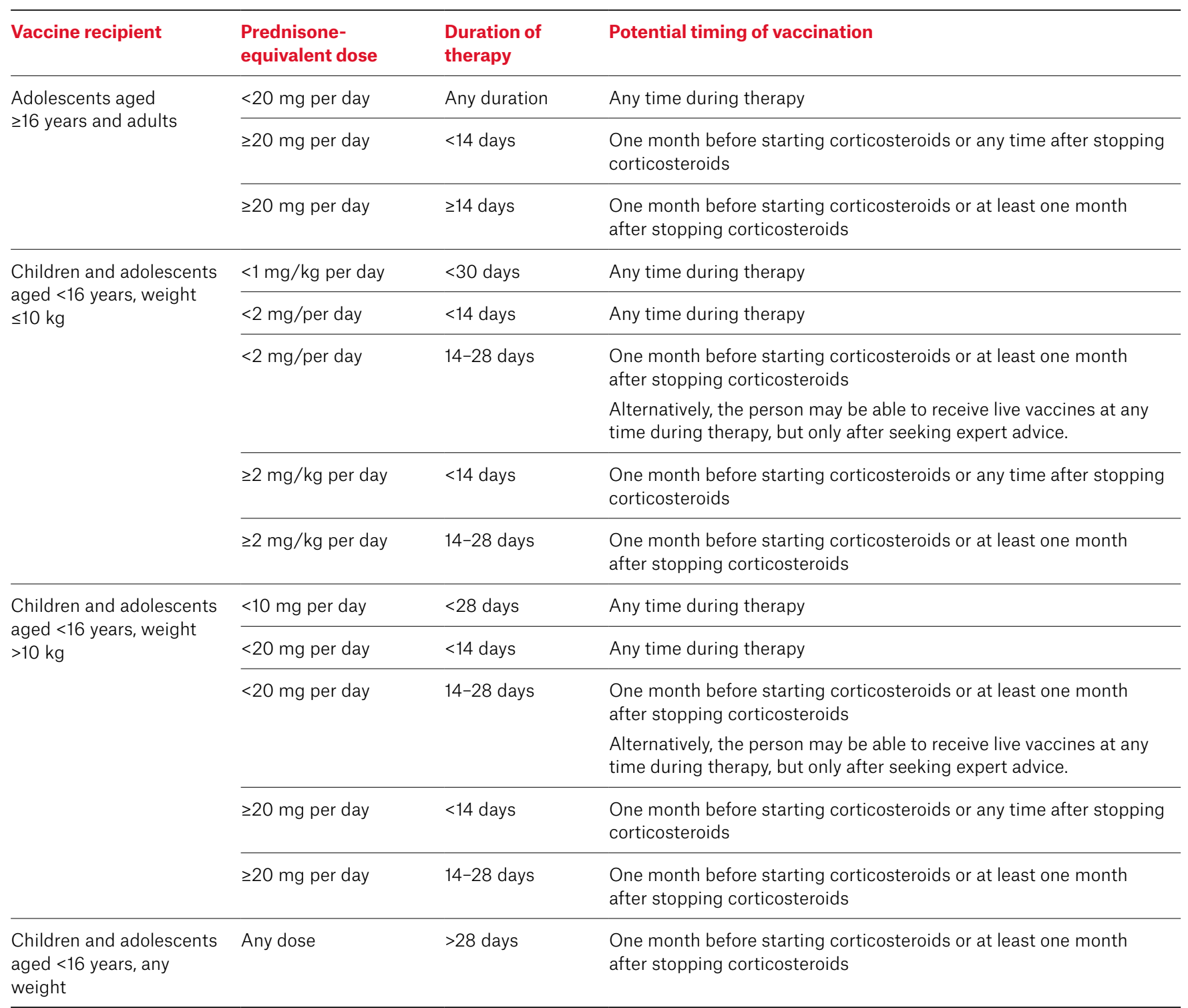

*This table assumes that the person has no other factors that contribute to their being immunocompromised (such as an underlying medical condition or use of other immunosuppressive medicines).

Reproduced with permission from The Australian immunisation handbook. Available at https://immunisationhandbook.health.gov.au/resources/handbooktables/table-recommended-timing-of-live-vaccine-doses-in-adults-and-children [Accessed 13 January 2020]. 
immunosuppression may predispose the person to more severe malaria should they become infected. Sun protection is also important, as some medications induce photosensitivity, and immunosuppression predisposes the patient to skin cancer.

\section{Planning for future immunosuppression}

For people with long-term conditions such as inflammatory rheumatic conditions, it may be possible to plan ahead for future travel and administer vaccines before commencing medications that cause significant immunocompromise. The Australian immunisation handbook recommends people receive all indicated live vaccines at least one month before starting immunosuppressive therapy. ${ }^{4}$ In the case of inactivated vaccines, these can be given up to two weeks before commencing immunosuppressive medication.

\section{Vaccination after withdrawal of immunosuppressant medication} Adding another layer of complexity is the fact that the period of time after discontinuation of immunosuppressive medication before a live vaccine can be administered varies between vaccines. The waiting times for vaccine administration after corticosteroids for a range of ages and dosage regimens are shown in Table 1. The general recommendation from The Australian immunisation handbook ${ }^{4}$ is that

\section{Table 2. Immunosuppressant medication and inactivated vaccines

\begin{tabular}{ll}
\hline Vaccine & Considerations \\
\hline Hepatitis A & For people taking medications that cause significant \\
immunocompromise, there is evidence that the response to a \\
single dose of hepatitis A vaccine will be inadequate, and two \\
doses over a six-month period should be administered before \\
travel. The US Centers for Disease Control and Prevention also \\
recommends giving immunoglobulin to provide passive immunity, \\
but this is not common practice in Australia.
\end{tabular}

Hepatitis B Hepatitis $B$ vaccination poses no additional risks for people taking immunosuppressant medication and is generally recommended. However, as is also the case with people undergoing dialysis, there may be an inadequate response to standard vaccination schedules, and a doubling of the usual dose may be considered. Serological testing to determine response is recommended.

\begin{tabular}{ll}
\hline Typhoid & $\begin{array}{l}\text { Oral typhoid vaccine should be avoided as it is a live vaccine, but } \\
\text { the inactivated parenteral Vi polysaccharide typhoid vaccine can } \\
\text { be used. }\end{array}$ \\
\hline Influenza & $\begin{array}{l}\text { Inactivated influenza vaccine is recommended but response may } \\
\text { be suboptimal. }\end{array}$ \\
\hline Pneumococcal & $\begin{array}{l}\text { Extra doses of pneumococcal vaccine may be indicated. The } \\
\text { Australian immunisation handbook recommends that adults with a } \\
\text { newly identified medical condition in Category A (which includes } \\
\text { immunosuppressive therapy) receive one lifetime dose of 13-valent } \\
\text { pneumococcal conjugate vaccine and three doses of 23-valent } \\
\text { pneumococcal conjugate vaccine. }\end{array}$ \\
\hline Rabies & $\begin{array}{l}\text { If pre-exposure rabies vaccination is needed, this should be } \\
\text { delivered using a standard intramuscular dosing regimen and not } \\
\text { intradermal vaccination. }\end{array}$ \\
\hline Meningococcal & $\begin{array}{l}\text { People taking the complement component inhibitor eculizumab } \\
\text { should be immunised against meningococcal disease with } \\
\text { MenACWY and, if } \geq 10 \text { years of age, MenB vaccine. They could also } \\
\text { consider antimicrobial prophylaxis. }\end{array}$ \\
\hline
\end{tabular}

people with rheumatic disease treated with biological or targeted synthetic diseasemodifying antirheumatic medications should not receive live vaccines until at least 12 months after therapy has ended. However, expert advice is often needed regarding the most appropriate interval for the person and their individual circumstances.

\section{Practicalities of travelling while taking immunosuppressant medication}

For people with chronic conditions, including those taking immunosuppressant medication, travel involves careful risk assessment and planning. Discussion and shared decision making with a GP and other health advisers will be needed. Travel health insurance, including medical evacuation insurance, is important but may be difficult to obtain as many policies have an exclusion for pre-existing conditions. Researching the health services available at the travel destination is part of the planning. Planning ahead is also important for travelling with medicines to ensure sufficient supplies and adequate documentation. ${ }^{6}$ In general, the Pharmaceutical Benefits Scheme allows up to a six-month supply of subsidised medications to be taken overseas for personal use. Medicines should be transported in their original packaging whenever possible and stored in cabin baggage rather than checked baggage. However, if the medicine is delivered via injection, prior approval from the airline will be needed. In general, the only documentation required by the airline is a doctor's letter. Refrigeration of medicines during flight is seldom necessary. Travellers should avoid purchasing medicines in low-income countries if possible, as substandard and counterfeit medicines are common. ${ }^{7}$

\section{Author}

Nicholas A Zwar MBBS, MPH, PhD, FRACGP, FACTM Executive Dean, Faculty of Health Sciences and Medicine, Bond University, Qld. nzwar@bond.edu.au Competing interests: None.

Funding: None.

Provenance and peer review: Commissioned, externally peer reviewed. 


\section{References}

1. Kotton CN, Kroger AT, Freedman DO.

Immunocompromised travellers. In: Brunette GW,

Nemhauser JB. CDC yellow book 2020: Health

information for international travel. New York:

Oxford University Press, 2017. p. 395-409.

2. Aw B, Boraston $S$, Botten D, et al. Travel medicine: What's involved? When to refer? Can Fam Physician 2014;60(12):1091-103.

3. Leder K, Steffen R, Cramer JP, Greenaway C. Risk assessment in travel medicine: How to obtain, interpret, and use risk data for informing pre-travel advice. J Travel Med 2015;22(1):13-20. doi: 10.1111/ jtm.12170.

4. Australian Technical Advisory Group on Immunisation (ATAGI). The Australian immunisation handbook. Canberra: Australian Government Department of Health, 2018.

5. Zwar N, Streeton CL. Pretravel advice and hepatitis A immunization among Australian travelers. J Travel Med 2007;14(1):31-36. doi: 10.1111/j.1708-8305.2006.00088.x.

6. Public Health England. Immunisation against infectious disease. London: Public Health England 2017.

7. Zwar N. Travelling with medicines in 2018. Aust Prescr 2018;41(4):102-04. doi: 10.18773/ austprescr.2018.034. 